\title{
Isolated Hepatic Basidiobolomycosis in a 2-Year-Old Girl: The First Case Report
}

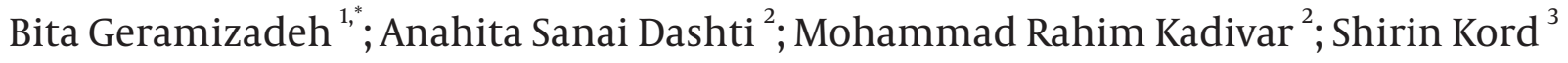 \\ ${ }^{1}$ Transplant Research Center, Pathology Department, Shiraz University of Medical Sciences, Shiraz, IR Iran \\ 2 Dr. Alborzi Microbiology Research Center, Department of Pediatrics, Shiraz University of Medical Sciences, Shiraz, IR Iran \\ 3 Department of Pathology, Shiraz University of Medical Sciences, Shiraz, IR Iran \\ *Corresponding Author: Bita Geramizadeh, Transplant Research Center, Pathology Department, Shiraz University of Medical Sciences, Shiraz, IR Iran. Tel: +98-9173143438, \\ E-mail: geramib@gmail.com
}

Received: May 20, 2015; Revised: June 28, 2015; Accepted: July 13, 2015

\begin{abstract}
Introduction: Gastrointestinal basidiobolomycosis is an emerging infection, with fewer than 80 cases reported in the English literature. Case Presentation: Also, a few cases of gastrointestinal basidiobolomycosis, accompanied by liver involvement as part of a disseminated disease, have been reported.

Conclusions: This is the first case report of an isolated liver involvement of this fungal infection in a 2-year-old girl, who presented with a liver mass resembling a hepatic abscess.

Keywords: Liver; Basidiobolomycosis; Immunocompetent
\end{abstract}

\section{Introduction}

Zygomycosis includes 2 orders, one of which causes fungal infections in an immunocompromised host (Mucorales) and the other in an immunocompetent host (Entomophthorales) (1).

Basidiobolus ranarum belongs to the second group and is a saprophyte found mostly in soil and decaying vegetable material (2). It is a low virulent fungus, and the first human case of this fungal infection was reported in 1956 in subcutaneous tissue (3). Since then, many cases of subcutaneous basidiobolomycosis have been reported. However, the gastrointestinal (GI) involvement of this fungus is an emerging infection and has rarely been reported (4). Recently, a few cases of GI basidiobolomycosis, accompanied by liver involvement as part of a disseminated disease, have been reported (5).

To the best of our knowledge, no case has been reported in the English literature with an isolated liver mass caused by basidiobolomycosis without the involvement of any other organ. Accordingly, herein we report our experience with a 2-year-old girl, who presented with a liver mass subsequently identified as basidiobolomycosis.

\section{Case Presentation}

A 2-year-old well-nourished and well-developed girl from the Iranian city of Kangan (Bushehr province) presented with vague and generalized abdominal pain. She was the first child of the family, born via normal vaginal delivery without any specific disorder. She had had a normal infancy until 2 months prior to her referral, when she developed abdominal pain with no response to routine treatment.

Physical examination was normal, except for mild hepatomegaly. Laboratory tests showed microcytic hypochromic anemia (hemoglobin level $=7.8 \mathrm{gr} / \mathrm{dL}$ ). White blood cell count was high (about 11-12000/cc) with significant eosinophilia (25\% - 35\%). Immunoserology tests revealed high C-reactive protein $(\mathrm{CRP}=13 \mathrm{mg} / \mathrm{L}$, normal $<6)$ and erythrocyte sedimentation rate $(E S R=83$, normal for the patient's age $<10$ ). Liver function tests showed high aspartate aminotransferase and alanine aminotransferase (57 and $45 \mathrm{IU} / \mathrm{L}$ respectively, normal < 31) and alkaline phosphatase $(4030 \mathrm{IU} / \mathrm{L}$, normal <300). Stool occult blood was performed 3 times, and the results were all negative.

Abdominal ultrasonography demonstrated a prominent liver with a well-defined mass lesion measuring $40 \times 35$ $\mathrm{cm}$. Another mass was detected in the hilar area. The other parts of the GI tract, including the stomach and intestine walls, were normal. Upper abdominal magnetic resonance imaging (MRI) showed normal thickness of the GI tract with no mass, but there were multiple masses in the liver. The first impression of both sonography and computed tomography scan was liver or biliary abscesses (Figure 1). TruCut needle biopsy displayed a mainly normal liver with foci of eosinophilic infiltration, which was nondiagnostic.

Copyright ( 2015, Kowsar Corp. This is an open-access article distributed under the terms of the Creative Commons Attribution-NonCommercial 4.0 International License (http://creativecommons.org/licenses/by-nc/4.0/) which permits copy and redistribute the material just in noncommercial usages, provided the original work is properly cited. 
Geramizadeh B et al.

Therefore, the patient underwent surgery, which showed multiple nonencapsulated liver masses with illdefined borders, the main one in the parenchyma and the other in the hilar area. During surgery, precise search was made to find any accompanied GI mass, but no mass was identified. Also, the omentum was completely free of any tumor or mass lesion. The masses were resected and sent for culture and pathologic studies. The pathology sections showed Splendore-Hoeppli bodies and many eosinophils as well as radiating eosinophilic granular material surrounding the fungal elements within the liver pa- renchyma and in the hilar mass within the lymph node tissue (Figures 2A and2B). The fungal elements exhibited broad hyphae with thin walls with no septae or sparse septation.

According to the characteristic pathologic features, the diagnosis of hepatic basidiobolomycosis was made. However, all the cultures including fungal and bacterial were negative. The immune system, cellular and humoral, of the patient was thoroughly investigated, even for the possibility of chronic granulomatous disease. All of the studies regarding the immune system were normal.
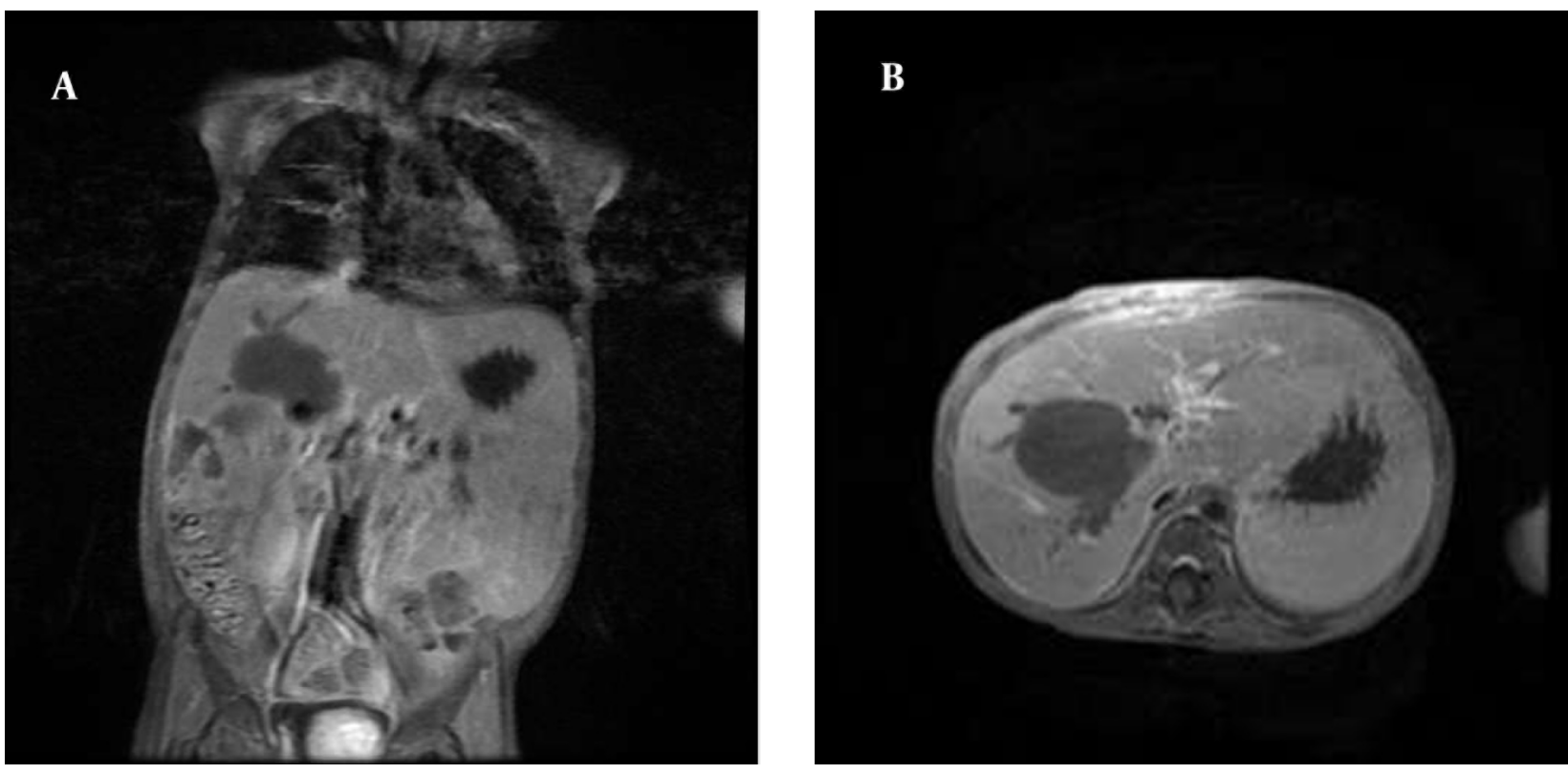

Figure 1. A, B: Magnetic Resonance Imaging of the Abdomen Shows Multiple Low-Signal Masses in the Liver Associated With Biliary Dilatation
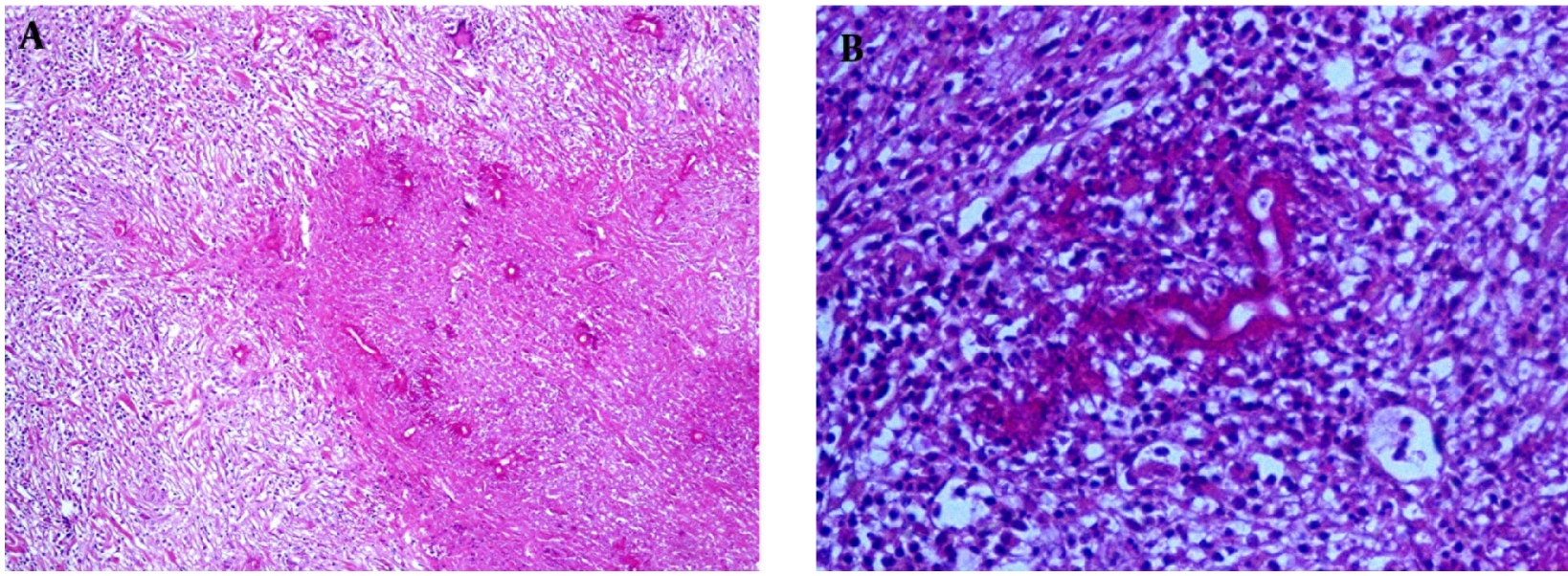

Figure 2. A, B: Degenerated Fungal Hyphae Surrounded by Granulomatous Reaction and Many Eosinophils in the Liver (2a: Low Power, 2b: High Power) 
Geramizadeh B et al.

Subsequently, antifungal therapy was started, mainly composed of amphotericin B (1 mg/ $\mathrm{kg} / \mathrm{d})$ for at least 6 months. Now after 3 months, the patient is well, the abdominal pain has been relieved, and also ESR and eosinophil counts have returned to normal level. She is still under treatment and follow-up.

\section{Discussion}

Basidiobolomycosis is an uncommon fungal infection caused by Basidiobolus ranarum, which is an environmental saprophyte (6). It causes human fungal infection in immunocompetent hosts. The most common reported site of involvement is subcutaneous tissue, caused by insect bite or contamination of wounds by soil and dust (7). However, GI involvement of this fungus is rare and seems to be secondary to the ingestion of contaminated food and water (8).

Fewer than 80 cases of GI basidiobolomycosis have been reported in the English literature(9), and a small number of them have been accompanied by a liver mass. These cases were reported from United States, Kuwait, Iraq, and Iran. The cases reported from Iran were from Shiraz, Tehran, and Mashhad (9). In our previous 14 cases, there was a wide variation of lifestyles, both from rural and urban areas (10). It means that the reported liver masses caused by basidiobolomycosis have always been part of a disseminated disease (10-13). To the best of our knowledge, there has been no report of an isolated liver mass caused by this pathogen without the involvement of the GI tract. The probable theory for the pathogenesis in isolated liver involvement can be the acquisition of this infection after the ingestion of contaminated material and dissemination of the fungus through small lymphatics in the GI tract without creating a mass in the intestine (11). The clinical presentation of hepatic basidiobolomycosis is very similar to tubular GI inasmuch as abdominal pain is the most common presenting symptom in the GI tract. Our patient also presented with abdominal pain (9).

Our patient underwent liver biopsy and then laparotomy with the clinical impression of malignancy, which is the exact usual preoperative diagnosis of GI basidiobolomycosis in the previous reports (13). The most important laboratory findings in our case were high ESR and CRP, with significant eosinophilia. Also aspartate aminotransferase, alanine aminotransferase, and alkaline phosphatase were significantly high, which were all indicative of parenchymal liver injury most probably secondary to an inflammatory or neoplastic process. The usual paraclinical findings in GI basidiobolomycosis are also the same (9).

Imaging studies play a key role in the diagnosis of liver masses before surgery or biopsy. All masses in the solid organs such as liver and kidney tend to show an inflammatory component with adjacent soft tissue stranding, with or without abscess formation (14). In this case, sonography and MRI were in favor of a liver mass; however, the possibility of a hepatic abscess was also considered.
In most of the previous cases of GI basidiobolomycosis, the final diagnosis was made after surgery and resection of the liver mass (9). Nonetheless, the gold standard for the diagnosis of every fungal infection is culture. In the majority of the previously reported cases of GI basidiobolomycosis, culture was either negative or was not performed because of the unavailability of the proper tissue (5). In our case, cultures turned out to be negative. The pathologic characteristics of this fungal infection are the presence of Splendore-Hoeppli bodies with many eosinophils and degenerated fungal hyphae (13). It can cause liver granuloma with heavy infiltration of eosinophilic liver granuloma and should be considered in the differential diagnosis of hepatic granulomas (15).

The best treatment in this pathogen is the resection of the mass, accompanied by antifungal therapy including itraconazole or amphotericin B. Our patient showed a dramatic response to amphotericin B. In less than a week, eosinophilia disappeared and ESR returned to normal and within 2 weeks, she resumed weight gain and her abdominal pain subsided. She is still under treatment, and the plan is to continue the antifungal therapy for at least 6 months, because our previous experience showed the high possibility of recurrence after an early discontinuance of the treatment.

In conclusion, basidiobolomycosis should be considered as a differential diagnosis of hepatic abscess with or without GI involvement to prevent delayed treatment.

\section{Authors' Contributions}

Bita Geramizadeh diagnosed the case and wrote and prepared the paper. Anahita Sanai Dashti handle the patient. Mohammad Rahim Kadivar treated the patient. Shirin Kord assisted in data collection.

\section{References}

1. Al-Shanafey S, AlRobean F, Bin Hussain I. Surgical management of gastrointestinal basidiobolomycosis in pediatric patients. $J$ Pediatr Surg. 2012;47(5):949-51.

2. Saadah OI, Farouq MF, Daajani NA, Kamal IS, Ghanem AT. Gastrointestinal basidiobolomycosis in a child; an unusual fungal infection mimicking fistulising Crohn's disease. J Crohns Colitis. 2012;6(3):368-72.

3. Kian Joe L, Pohan A, Tjoei Eng NI, Van Der Meulen H. Basidiobolus ranarum as a cause of subcutaneous mycosis in Indonesia. AMA Arch Derm. 1956;74(4):378-83.

4. Geramizadeh B, Modjalal M, Nabai S, Banani A, Forootan HR, Hooshdaran F, et al. Gastrointestinal zygomycosis: a report of three cases. Mycopathologia. 2007;164(1):35-8.

5. Ejtehadi F, Anushiravani A, Bananzadeh A, Geramizadeh B. Gastrointestinal basidiobolomycosis accompanied by liver involvement: a case report. Iran Red Crescent Med J. 2014;16(9):e14109.

6. Nguyen BD. CT features of basidiobolomycosis with gastrointestinal and urinary involvement. AJR Am J Roentgenol. 2000;174(3):878-9.

7. Khan ZU, Khoursheed M, Makar R, Al-Waheeb S, Al-Bader I, AlMuzaini A, et al. Basidiobolus ranarum as an etiologic agent of gastrointestinal zygomycosis. J Clin Microbiol. 2001;39(6):2360-3.

8. Khan ZU, Chugh TD. Invasive fungal infections in Kuwait: A retrospective study. Indian J Chest Dis Allied Sci. 2000;42(4):279-87.

9. Geramizadeh B, Heidari M, Shekarkhar G. Gastrointestinal Basidiobolomycosis, a Rare and Under-diagnosed Fungal Infection 
in Immunocompetent Hosts: A Review Article. Iran J Med Sci. 2015;40(2):90-7.

10. Geramizadeh B, Foroughi R, Keshtkar-Jahromi M, Malek-Hosseini SA, Alborzi A. Gastrointestinal basidiobolomycosis, an emerging infection in the immunocompetent host: a report of 14 patients. J Med Microbiol. 2012;61(Pt 12):1770-4.

11. Hassan HA, Majid RA, Rashid NG, Nuradeen BE, Abdulkarim QH, Hawramy TA, et al. Eosinophilic granulomatous gastrointestinal and hepatic abscesses attributable to basidiobolomycosis and fasciolias: a simultaneous emergence in Iraqi Kurdistan. BMC Infect Dis. 2013;13:91.

12. van den Berk GE, Noorduyn LA, van Ketel RJ, van Leeuwen J, Be- melman WA, Prins JM. A fatal pseudo-tumour: disseminated basidiobolomycosis. BMC Infect Dis. 2006;6:140.

13. Vikram HR, Smilack JD, Leighton JA, Crowell MD, De Petris G. Emergence of gastrointestinal basidiobolomycosis in the United States, with a review of worldwide cases. Clin Infect Dis. 2012;54(12):1685-91.

14. Flicek KT, Vikram HR, De Petris GD, Johnson CD. Abdominal imaging findings in gastrointestinal basidiobolomycosis. Abdom Imaging. 2015;40(2):246-50.

15. Geramizadeh B, Jahangiri R, Moradi E. Causes of hepatic granuloma: a 12-year single center experience from southern Iran. Arch Iran Med. 2011;14(4):288-9. 